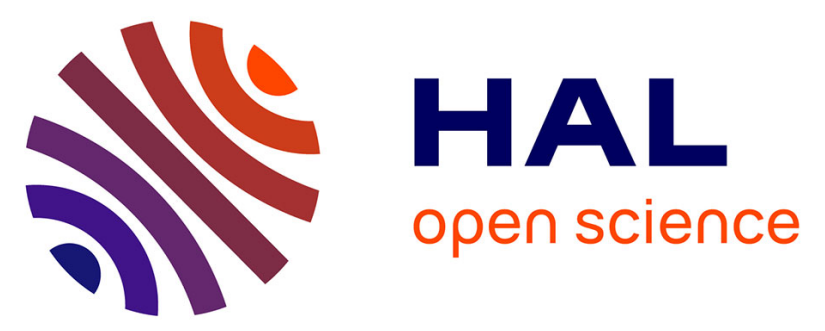

\title{
La dernière phase glaciaire du haut bassin de la Fecht (Vosges, France) : dynamique de l'englacement et chronologie relative de la déglaciation
}

Rémi Andréoli, Thierry Rosique, Michael Schmidt, Jean-Michel Carozza

\section{To cite this version:}

Rémi Andréoli, Thierry Rosique, Michael Schmidt, Jean-Michel Carozza. La dernière phase glaciaire du haut bassin de la Fecht (Vosges, France): dynamique de l'englacement et chronologie relative de la déglaciation. Géomorphologie: relief, processus, environnement, 2006, Varia, 12 (1), pp.23-36. 10.4000/geomorphologie.455 . hal-02562665

\section{HAL Id: hal-02562665 https://hal.science/hal-02562665}

Submitted on 4 May 2020

HAL is a multi-disciplinary open access archive for the deposit and dissemination of scientific research documents, whether they are published or not. The documents may come from teaching and research institutions in France or abroad, or from public or private research centers.
L'archive ouverte pluridisciplinaire $\mathbf{H A L}$, est destinée au dépôt et à la diffusion de documents scientifiques de niveau recherche, publiés ou non, émanant des établissements d'enseignement et de recherche français ou étrangers, des laboratoires publics ou privés. 


\section{Géomorphologie : relief, processus,}

environnement

vol. 12 - $\mathrm{n}^{\circ} 1 \mid 2006$

Varia

\section{La dernière phase glaciaire du haut bassin de la Fecht (Vosges, France) : dynamique de l'englacement et chronologie relative de la déglaciation}

The last glacial epoch in the upper Fecht basin (Vosges, France): past glacial dynamics and relative chronology of glacier retreat

Rémi Andreoli, Thierry Rosique, Michaël Schmidt et Jean-Michel Carozza

\section{OpenEdition \\ Journals}

Édition électronique

URL : http://journals.openedition.org/geomorphologie/455

DOI : $10.4000 /$ geomorphologie.455

ISSN : 1957-777X

Éditeur

Groupe français de géomorphologie

Édition imprimée

Date de publication : 1 avril 2006

Pagination : 23-36

ISSN : 1266-5304

Référence électronique

Rémi Andreoli, Thierry Rosique, Michaël Schmidt et Jean-Michel Carozza, «La dernière phase glaciaire du haut bassin de la Fecht (Vosges, France) : dynamique de l'englacement et chronologie relative de la déglaciation », Géomorphologie : relief, processus, environnement [En ligne], vol. 12 - n 1 | 2006, mis en ligne le 01 avril 2008, consulté le 28 avril 2019. URL : http://journals.openedition.org/ geomorphologie/455 ; DOI : 10.4000/geomorphologie.455 


\title{
La dernière phase glaciaire du haut bassin de la Fecht (Vosges, France) : dynamique de l'englacement et chronologie relative de la déglaciation
}

\section{The last glacial epoch in the upper Fecht basin (Vosges, France): past glacial dynamics and relative chronology of glacier retreat}

\author{
Rémi Andreoli*, Thierry Rosique*, Michaël Schmidt*, Jean-Michel Carozza**
}

\begin{abstract}
Résumé
L'étude stratigraphique et sédimentologique des dispositifs glaciaires de retrait d'un bassin des Vosges centrales - haut bassin de la Fecht - permet de proposer un schéma cohérent de la dernière phase d'englacement, du dernier maximum glaciaire (« stade de Metzeral ») à la déglaciation définitive du massif. Le retrait glaciaire s'est déroulé en quatre grandes étapes. Le mouvement global de décrue est interrompu par un épisode bref de récurrence glaciaire, signé par le complexe frontal de la moyenne vallée de la Wormsa (stade 3).
\end{abstract}

Mots clés : Vosges, Fecht, moraines, dépôts fluvioglaciaires, stades glaciaires, dernier maximum glaciaire, déglaciation.

\begin{abstract}
Based on stratigraphical and sedimentological studies of glacial and fluvioglacial deposits in the Fecht catchment (central Vosges), this work reconstructs the pattern of ice retreat from the Last Glacial Maximum (LGM, "stade de Metzeral") to the definitive deglaciation of the Vosges mountains. We also propose a relative chronology involving four stages, in which retreat was interrupted by a brief glacial readvance represented by the stage 3 terminal moraines in the middle section of the Wormsa valley.
\end{abstract}

Key words: Vosges mountains, Fecht River, glacial deposits, fluvioglacial deposits, glacial stages, Last Glacial Maximum, glacial retreat.

\section{Abridged English Version}

In the Vosges mountains, both the number and forms of glaciation are controversial, particularly with respect to the last glacial advance (Seret, 1967, 1990; Mercier and Jeser, 2001, 2004; Flageollet, 2002). This study focuses on the Fecht glacial subsystem in the Metzeral area (eastern central part of the Vosges mountains) and proposes a consistent explanation, divided into four stages describing glacier fluctuations from the Last Glacial Maximum to the definitive retreat of ice from the upper Fecht drainage basin. In this area, two groups of tills and moraines are distinguished (fig. 1): an incomplete till system termed group I, probably of Rissian age according to J. Tricart, 1963; and Last
Glacial Maximum tills and moraines belonging to group II. Group II includes tills and discontinuous lateral moraines of the middle section of the trunk valley (IIa). They originate from a first maximal glacial advance which, according to the work of J.-L. Mercier and N. Jeser $(2001,2004)$ and based on analogies that are found of the same formations on the western part of the Vosges mountains (Seret, 1967, 1990; Jeser, 2000; Mercier and Jeser, 2001, 2004), probably occurred in middle Würm times. Group II also includes tills and terminal moraines of the last glacial advance recorded in the upper valley reaches $(I I b)$. The terminal moraines of the last glacial advance (group IIb) can be subdivided into four stages (fig. 1). The Last Glacial Maximum (LGM) in

\footnotetext{
* Faculté de Géographie et d'Aménagement de l'université Louis Pasteur, 3 rue de l'Argonne, 67083 Strasbourg. E-mail : andreoli_remi@yahoo.fr ; rosique@equinoxe.u-strasbg.fr

** GEODE-UMR 5602 CNRS, Faculté de Géographie et d'Aménagement de l'université Louis Pasteur, 3 rue de l'Argonne, 67083 Strasbourg cedex.

E-mail : jmcarozza@yahoo.fr
} 
Metzeral is represented by a lateral and terminal morainic complex and a fluvioglacial terrace $T_{1 a}$ (stages $1 a$ and $1 b$ ). Terminal moraines of stage $2(2 a, 2 b$ and $2 c)$ are found 3.5 kilometres upstream of Metzeral, near Erbersch. Stage 3 terminal moraines $(3 a$ and $3 b)$ are generally located in glacial tributary basins of the main valleys (Seestaedtle, Kolbenfecht and Wormsa basins). Finally, ice persisted in glacial cirques beneath the principal mountain ridge, leaving well preserved tills and moraines (stage 4, "stade des cirques") before ice retreat became definitive. An outcrop of glacial and fluvioglacial deposits of stage 3 in the middle section of the Wormsa valley exhibits, from bottom to top, fluvioglacial sands and gravels topped by a till. The lithostratigraphic succession ends with fluvioglacial and glaciolacustrine sediments. This exposure suggests a first stage of ice retreat probably occurring after stage 2 (proglacial sands and gravels) followed by a short glacial advance (till, stage 3a). Subsequently, the beginning of ice retreat in the middle section of the Wormsa valley led to the sedimentation of fluvioglacial and glacio-lacustrine deposits in the basin between the ice margin and the terminal moraine $3 a$. Terminal moraine $3 b$ is probably coeval with the upper part of the fluvioglacial and glacio-lacustrine deposits and it corresponds to the last position of the ice tongue in the middle section of the Wormsa valley before ice retreated to the glacial cirques. During the Last Glacial Maximum, ice streams of the upper part of the Fecht drainage basin formed short alpine glaciers (ice tongue 7 kilometres in length in the Grande Fecht valley). To summarize, ice retreat occurred in four main stages: a first step of glacial tongue separation (stage 2); a rapid phase of glacial retreat broken by a brief glacial advance represented by the stage 3 end moraines; finally, ice persisted in glacial cirques (stage 4) before disappearing entirely from the landscape. The chronology here is relative, with no absolute dating involved. Some uncertainty therefore remains regarding the age of definitive ice retreat in the Fecht Basin in particular, and more generally throughout the Vosges mountains. However, this study brings into sharper focus a new key area of glacial activity in the Vosges mountains. The detailed mapping (figs 1 to 4) and relative chronology (fig. 7) provide a frame for conducting coherent sampling strategies for ongoing and future radiometric studies.

\section{Introduction}

Les travaux conduits sur les dépôts morainiques de la retombée sud-ouest du massif vosgien ont cerné généralement le déroulement des phases successives d'englacement de cette partie de la chaîne au cours du Quaternaire récent (Seret, 1967 ; Seret et al., 1990). Toutefois, les tentatives de synthèse régionale sont rares (Flageollet, 2002 ; Mercier et Jeser, 2001, 2004 ; Jeser, 2000) et, à l'échelle du massif vosgien, le nombre de glaciations et les modalités précises de la dynamique glaciaire divergent selon les auteurs. La présence de plusieurs calottes durant les glaciations du Saalien et du Weichselien moyen ne semble plus faire aucun doute aujourd'hui (Seret et al., 1990 ; Flageollet, 2002).
Mais les modalités de la dernière glaciation divergent suivant les auteurs : G. Seret (1967) puis J.-C. Flageollet (2002) proposent une glaciation de vallée complexe présentant de multiples langues de glaces divergentes : glaciers de vallée ramifiés (Flageollet, 2002) ; glaciers anastomosés selon J. Tricart (1963) décrivant les derniers appareils du massif du Hohneck. N. Jeser (2000), J.-L. Mercier et N. Jeser (2001) envisagent une séparation en plusieurs petites calottes indépendantes s'individualisant en glaciers de vallée lors de la déglaciation.

Sur le versant oriental du massif vosgien, les héritages morainiques constituent un trait dominant des paysages géomorphologiques des hautes vallées. S'ils sont souvent remarquables par leur fraîcheur de forme, ils peuvent offrir parfois une morphologie ambiguë qui rend leur interprétation délicate. Dans ce domaine, les limites spatiales des différentes phases d'englacement, l'âge du Dernier Maximum Glaciaire (DMG) et les conditions du retrait définitif des langues de glaces restent largement méconnus. Des corrélations ont été néanmoins tentées entre les dispositifs morainiques des versants occidental et oriental des Vosges (Flageollet, 2002 ; Mercier et Jeser, 2001, 2004 ; Jeser, 2000). Il faut pourtant admettre que celles-ci sont incertaines du fait de l'absence de continuité dans l'agencement des formes par-delà la crête faîtière et l'absence de référent chronologique absolu à l'échelle du massif.

Dans ce contexte, nos recherches (Andreoli, 2003) se sont concentrées sur le sous-système glaciaire d'une vallée orientale des Hautes Vosges, dont la morphologie glaciaire a souvent été prise pour exemple (Baulig, 1922 ; Sittig, 1933 ; Krittlé, 1956 ; Tricart, 1963) : le haut bassin de la Fecht en amont de Munster. En complément des premiers travaux réalisés par M. Schmidt (2002), les données géomorphologiques, sédimentologiques et stratigraphiques réunies permettent de préciser les modalités de la dernière phase d'englacement et les premières étapes de la récession glaciaire. Le schéma morphodynamique et la chronologie relative proposés sont volontairement restreints à l'échelle locale du haut bassin de la Fecht. Cet apport ne constitue, par ailleurs, qu'une étape préliminaire, prolongée actuellement par la recherche de marqueurs chronologiques absolus dans les divers stades de retrait reconnus et qui s'inscrit dans le débat ouvert par les résultats des analyses cosmogéniques $\left({ }^{10} \mathrm{Be}\right)$ obtenus récemment dans la vallée de la Wormsa (Mercier et al., 1999 ; Bourlès et al., 2004).

\section{Le haut bassin de la Fecht : l'empreinte des phénomènes glaciaires}

Les vallées de la Fecht, Grande Fecht et Petite Fecht appartiennent au vaste bassin de la Fecht. Celui-ci s'ouvre à l'est de la crête faîtière des Vosges, dans le massif du Hohneck (1 363 m). Dans ce secteur, la crête, allongée nord-sud à des altitudes toujours supérieures à $1300 \mathrm{~m}$, est ouverte par deux cols d'altitude inférieure à $1200 \mathrm{~m}$ (col de la Schlucht et Firstmiss). Le versant oriental du massif est 
abrupt (rochers de la Martinswand, arête des Spitzkoëpfe) et les cours d'eau drainent de profondes vallées en auge.

Le haut bassin de la Fecht est intégralement façonné dans les roches du socle cristallin (essentiellement granites antéhercyniens et hercyniens précoces, granites intrusifs ; schistes et grauwackes viséens localement). Il présente, d'autre part, des modelés et dépôts variés, intimement liés aux glaciations quaternaires. Ainsi, la section amont du bassin de la Grande Fecht, comprise entre la crête faîtière et la localité de Metzeral, concentre les principaux héritages glaciogéniques. La moyenne vallée de la Fecht, de Metzeral à Munster, est dominée par des remblaiements fluvioglaciaires et alluviaux qui s'organisent en terrasses emboîtées : moyennes terrasses fluvioglaciaires $T_{1 a}, T_{1 b}$ et $T_{1 c}$ et basse terrasse postglaciaire $\mathrm{T}_{0}$ (Andreoli, 2003).
Les caractéristiques géomorphologiques, la fraîcheur relative des formes et la position des héritages glaciaires permettent de différencier deux ensembles morainiques (fig. 1). Le groupe I correspond à des dépôts morainiques mal connus, empâtant certains vallons affluents des vallées principales dans les sections moyennes. Leur morphologie, peu caractéristique, est due à leur fréquent remaniement par la solifluxion sur les versants ou par les ruisseaux dans les talwegs actuels. Ces formations tapissent notamment le vallon de Breitenbach, affluent de la Fecht. Selon J. Tricart (1963), ces héritages pourraient constituer des moraines résiduelles associées aux glaciers du « Riss ». Le groupe II comprend des complexes morainiques de vallées bien construits (moraines latérales et frontales) que nous subdivisons en deux sous-ensembles : groupe IIa et IIb.

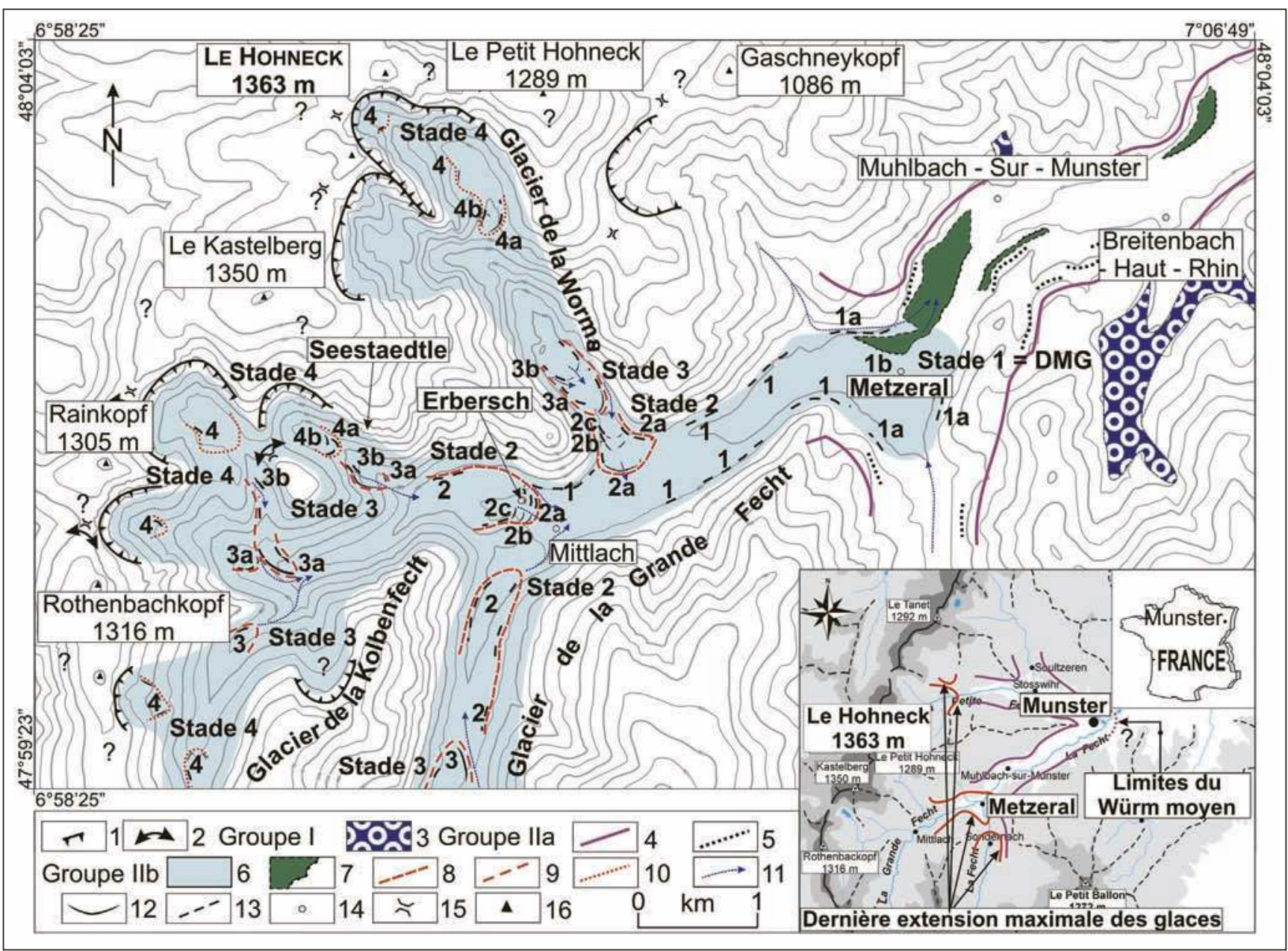

Fig. 1 - Chronologie relative de la déglaciation de la dernière récurrence glaciaire 1 : principaux cirques ; 2 cols de transfluence ; 3 : dépôts morainiques anciens (groupe I) ; 4 : limites de l'extension au Würm moyen ; 5 : dépôts morainiques lacunaires des sections moyennes (groupe Ila) ; 6 : dernière extension maximale des glaces (stade de Metzeral) $; 7:$ moyenne terrasse fluvioglaciaire supérieure T1a ; $8:$ limites du stade 2 (stade de Erbersch) ; 9 : limites du stade 3 (stade de Seestaedtle) ; 10 : limites du stade 4 (stade des cirques) ; 11 : principaux chenaux juxtaglaciaires et proglaciaires ; 12 : constructions morainiques frontales du groupe Ilb ; 13 : cordons morainiques discontinus ; 14 : localité ; 15 : col ; 16 : sommet.

Fig. 1 - Relative chronology of ice retreat since the Last Glacial Maximum. 1: principal glacial cirques; 2: ice pass; 3: old till (group I); 4: glacial extent during Middle Würmian times; 5: poorly preserved tills of the middle valley sections (group Ila); 6: ice extent during the Last Glacial Maximum (Metzeral stage); 7: fluvioglacial terrace T1a ; 8: glacial extent of the "Erbersch » stage (2); 9: glacial extent of the "Seestaedtle" stage (3); 10: glacial extent of the cirques stage (4); 11: principal proglacial or juxtaglacial streams; 12: frontal morainic bar of group Ilb; 13: discontinuous morainic ridges; 14: locality; 15: pass;16: summit. 
Les constructions morainiques lacunaires des sections moyennes des vallées (groupe IIa) forment des cordons morainiques latéraux discontinus disposés sur les versants des sections moyennes des vallées. Ils s'ordonnent parfois en faisceaux regroupant deux, voire trois niveaux, perchés à 20-30 m au-dessus du fond actuel des vallées. Latéralement, ces formations glaciogéniques s'associent à des dépôts d'obturation latérale développés aux débouchés des vallons affluents des vallées principales, notamment, dans la vallée de la Fecht en amont de Munster, à Muhlbach-surMunster, Breitenbach et Luttenbach. Les crêtes morainiques résiduelles des moyennes vallées semblent avoir des équivalents sur les versants des hauts bassins montagnards, où des placages morainiques sont perchés à 10-20 m au-dessus des fonds de talweg. Il faut admettre, cependant, que le raccord géométrique est ici incertain car ces vestiges résiduels sont remaniés sur les versants. Ce type d'héritage, bien visible à l'affleurement dans le secteur de Saegmatt (versant ubac de la Petite-Fecht), comporte des blocs de granite beaucoup plus altérés (blocs arénisés et altération de surface) que les dépôts de fond de vallée.

Dans les hauts bassins montagnards, en amont de Metzeral dans la vallée de la Grande Fecht ou de Grossmatten dans la vallée de la Petite Fecht, les accumulations morainiques de fond de vallée constituent de belles formes construites. Les dispositifs comprennent des complexes morainiques frontaux (rides morainiques et épandages proglaciaires), obturant tout ou partie des vallées, et des cordons morainiques latéraux plus ou moins continus au pied des versants. Ces dépôts présentent un état de fraîcheur remarquable : les blocs de granites sont sains, les sols développés sur ces formations sont généralement peu évolués. Les héritages ainsi décrits correspondent aux formations morainiques les plus récentes du haut bassin de la Fecht, édifiées lors de la dernière progression glaciaire. Ils apparaissent dès lors au cœur de la problématique de la présente étude.

\section{Les dispositifs de retrait de la dernière phase d'englacement}

Les complexes morainiques du groupe IIb qui jalonnent le haut bassin de la Grande Fecht s'organisent spatialement en quatre dispositifs glaciaires latéro-frontaux bien distincts. Quatre stades se trouvent ainsi définis d'aval en amont de la vallée : stade 1 de Metzeral, 2 de Erbersch, 3 de Seestaedtle et stade 4 des constructions des cirques d'altitude (fig. 1). Ce système glaciaire présente d'importantes similitudes avec celui de la haute vallée de la Petite Fecht (Andreoli, 2003). Le tableau 1 résume les corrélations que nous proposons entre les moraines des stades des deux vallées.

\section{Stade 1 « de Metzeral »}

À Metzeral l'extension des glaces est soulignée par des cordons morainiques latéraux ou latéro-frontaux quasicontinus, se prolongeant sur plusieurs kilomètres en amont de la localité (fig. 2). Emboîtée en contrebas des constructions morainiques juxtaglaciaires, une vaste terrasse fluvio- glaciaire $\left(\mathrm{T}_{1 \mathrm{a}}\right)$ se développe directement en aval de Metzeral et se prolonge dans la vallée de la Fecht, jusqu'à Muhlbachsur-Munster. Celle-ci présente un faciès proglaciaire caractéristique (alluvions hétérométriques comprenant des blocs de taille souvent supérieure à un mètre cube). Le dispositif est complété d'autre part par deux niveaux de terrasses secondaires (nappes fluvioglaciaires de retrait $\mathrm{T}_{1 \mathrm{~b}}, \mathrm{~T}_{1 \mathrm{c}}$ ), échelonnées rapidement dans la gorge fluviatile ouverte dans la terrasse $\mathrm{T}_{1 \mathrm{a}}$. Enfin, dans ce bassin situé à la confluence des vallées de la Fecht et de la Grande Fecht, le stationnement des glaces s'est accompagné de la mise en place de dépôts glaciolacustres et fluvioglaciaires d'obturation latérale (formations de la colline de l'Emme dans la vallée de la Fecht d'après F. Ménillet et al., 1976 : notice de la carte géologique au 1/50 000, $\mathrm{n}^{\circ} 377$, Munster). Ces données suggèrent une évolution en trois étapes : a) un maximum de progression glaciaire signé par des complexes morainiques latérofrontaux résiduels (stade 1a, fig. 1). Dans la basse vallée de la Grande Fecht, les glaces débordaient largement sur leur rive droite, obturant ainsi les apports hydrosédimentaires provenant du haut bassin de la Fecht. b) un stade rapproché de décrue glaciaire (stade 1b, fig. 1). Les écoulements d'eau de fusion glaciaire ont alors incisé les moraines du DMG puis accumulé les alluvions proglaciaires de $\mathrm{T}_{1 \mathrm{a}}$. c) un retrait saccadé des langues glaciaires vers leur haute vallée, responsable de l'évacuation d'une grande partie des édifices morainiques terminaux et du façonnement des terrasses $\mathrm{T}_{1 \mathrm{~b}}$ et $\mathrm{T}_{1 \mathrm{c}}$ (vallée de la Grande Fecht).

Le complexe morainique frontal de Metzeral possède un homologue aux caractéristiques similaires dans la vallée de la Petite Fecht à Grossmatten. En effet, la partie centrale de l'édifice morainique terminal n'a pas été préservée de l'érosion postérieure. L'extension des glaces jusqu'à Grossmatten est signalée par la présence de cordons continus de moraines latérales. À Grossmatten, la moraine est partiellement recouverte par un vaste épandage torrentiel alors qu'elle s'infléchit vers le centre de la vallée en moraine latéro-frontale. L'ensemble du dispositif est complété par un mince lambeau de terrasse en continuité géométrique avec la moyenne terrasse supérieure $\mathrm{T}_{1 \mathrm{a}}$ de la vallée de la Petite Fecht (Andreoli, 2003).

\section{Stade 2 « de Erbersch »}

Dans la vallée de la Grande Fecht, les moraines du stade 2 se situent à 3,5 km en amont du dispositif de Metzeral. Celles-ci se développent à la confluence de deux vallées affluentes (Kolbenfecht, vallon de la Wormsa). Dans le bassin de la Petite Fecht, les héritages morainiques du stade 2 sont situés directement en amont des moraines du DMG. Ils correspondent dans ce secteur à un barrage morainique complexe, issu de la rencontre de deux langues glaciaires (Petite Fecht et Gaschney) au lieu dit Saegmatt.

Généralement, ces dispositifs présentent des caractéristiques analogues (fig. 1 et tab. 1); le complexe morainique frontal de Erbersch à Mittlach (Jeser, 2000 ; Schmidt, 2002 ; Flageollet, 2002 ; Mercier et Jeser, 2004) dans la vallée de la Kolbenfecht en constitue le plus bel exemple. Situé 


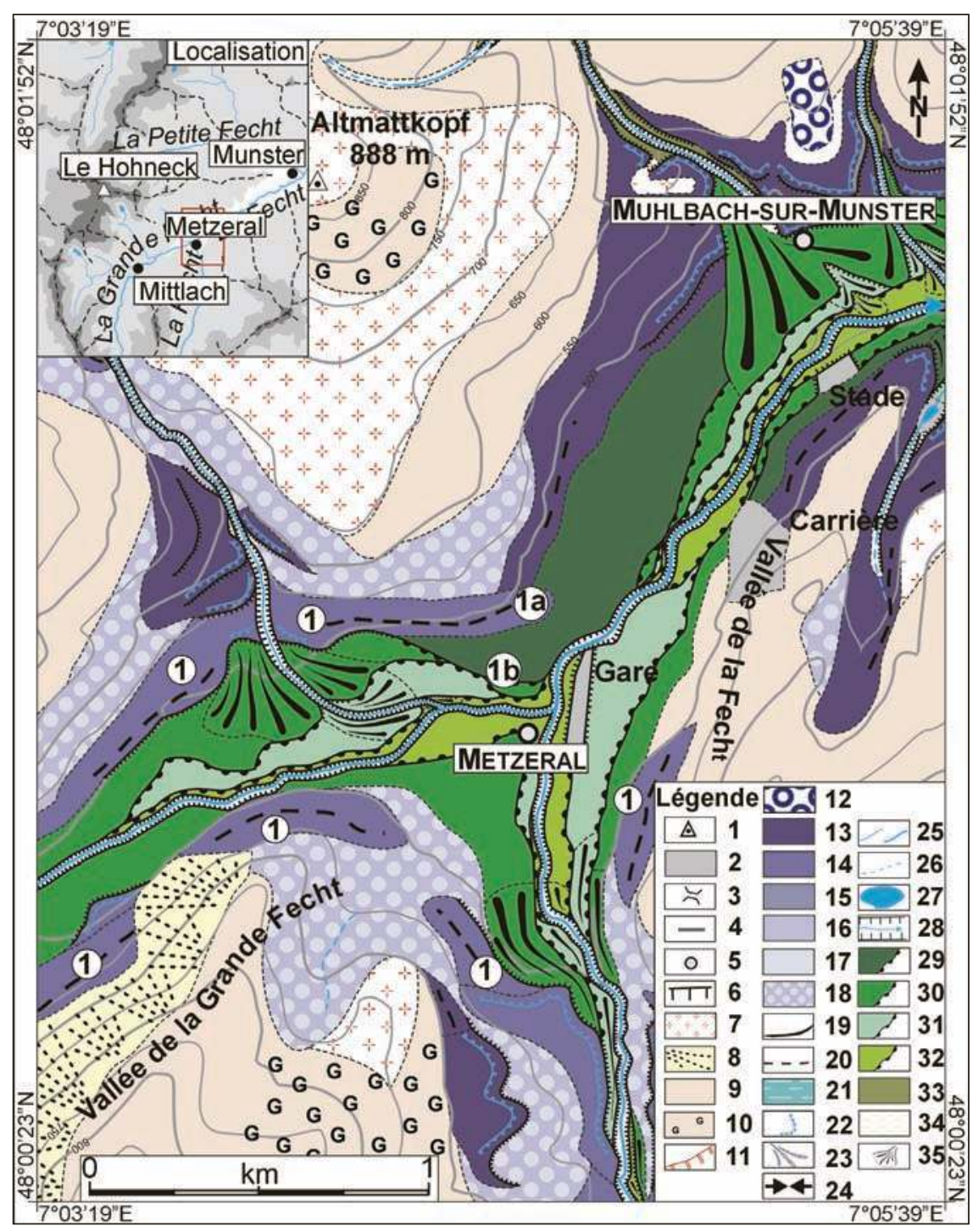

Fig. 2 - Carte des formations superficielles de Metzeral (Vosges, France). 1 : sommet ; 2 : remblais anthropiques ; 3 : col ; 4 : barrage ; 5 : localité $; 6$ : talus $; 7$ : arènes granitiques $; 8$ : éboulis à gros blocs ; 9 : formations de pente périglaciaires indéterminées ou polygéniques ; 10 : gélifluxion ; 11 : escarpement ; 12 : moraines « rissiennes »;13: moraines du Würm moyen ; 14 : moraine (stade 1) ; 15 : moraines (stade 2) ; 16 : moraines (stade 3) ; 17 : moraines (stade 4) ; 18 : dépôt morainique remanié ; 19 : ride morainique ; 20 : cordon morainique discontinu ; 21 : dépôt fluvioglaciaire et glacio-lacustre de retrait ; 22 : dépôt d'obturation latérale, gradin latéral ou kame ; 23 : cône proglaciaire ; 24 : verrou ; 25 : cours d'eau pérenne ; 26 cours d'eau intermittent ; 27 : lac, étang ; 28 : lit majeur actuel ; 29 : moyenne terrasse supérieure $\mathrm{T} 1 \mathrm{a} ; 30$ : moyenne terrasse intermédiaire $\mathrm{T} 1 \mathrm{~b} ; 31$ : moyenne terrasse inférieure T1c ; 32 : basse terrasse postglaciaire T0 ; 33 : dépôt torrentiel ; 34 : tourbière, marais ; 35 : cône de déjection.

Fig. 2 - Map of Metzeral field (Vosges, France). 1: summit; 2: backfill; 3: pass; 4: dam; 5: locality; 6: bank; 7: grus; 8: bouldery talus; 9: undetermined or polygenetic periglacial deposits; 10: gelifluxion; 11: steep slope; 12: till (Riss); 13: till (middle Würm); 14: stage 1 till; 15: stage 2 till; 16: stage 3 till; 17: stage 4 till; 18: reworked till; 19: morainic ridge; 20 : discontinuous morainic ridge; 21: fluvioglacial and glaciolacustrine deposits indicating ice retreat; 22: ice-contact valley-side terrace, or kame delta; 23: proglacial fan; 24: rock-bar; 25: river; 26 ephemeral stream; 27: lake, pond; 28: present-day river bed; 29: upper fluvioglacial terrace T1a; 30: fluvioglacial terrace T1b; 31: fluvioglacial terrace T1c; 32: postglacial terrace T0; 33: torrential deposits; 34: peat bog; 35: alluvial or torrential fan. à l'aval du vallon de la Kolbenfecht, ce dernier obture la vallée de la Grande Fecht sur toute sa largeur. Les accumulations morainiques sont puissantes et forment trois rides successives $(2 \mathrm{a}, 2 \mathrm{~b}, 2 \mathrm{c})$. Ce dispositif s'associe latéralement à des cordons morainiques latéraux discontinus et se prolonge, à l'aval, par un petit épandage proglaciaire résiduel. Ajoutons, enfin, que deux cônes fluvioglaciaires de retrait sont emboîtés dans les dépôts morainiques latéro-frontaux.

\section{Stade 3 « de Seestaedtle »}

Les accumulations morainiques appartenant au stade 3 se situent dans les ombilics ou en aval des gorges de raccordement des bassins élémentaires affluents des vallées principales (fig. 1 et tab. 1). Dans le haut bassin de la Grande Fecht, ces dispositifs caractérisent ainsi les vallées de la Kolbenfecht, de Seestaedtle, de la Wormsa et de la Grande Fecht de Mittlach. Dans la vallée de la Petite Fecht, les moraines du stade 3 clôturent l'ombilic de Rothried. Les héritages morainiques présentent certaines disparités entre les vallons ; les dynamiques de sédimentation relevaient, en effet, d'appareils glaciaires élémentaires dont le fonctionnement était inféodé aux contraintes topoclimatiques (exposition notamment) et géomorphologiques locales (superficie de la zone d'alimentation, pente et profil transversal de la vallée).

Toutefois, deux arcs morainiques successifs (3a et $3 b$ ) s'individualisent nettement dans les vallons de Seestaedtle, de la Kolbenfecht et de la Wormsa. Ceux-ci sont associés à des cônes proglaciaires d'importance réduite, disparaissant rapidement vers l'aval. Le dispositif glaciaire et fluvioglaciaire de la vallée de la Wormsa permet d'appréhender la complexité de ce stade et fait l'objet d'une analyse plus approfondie dans cet article.

\section{Stade 4 : les constructions morainiques des cirques}

Les glaciers ont persisté en position d'abri dans les cirques glaciaires des hauts bassins montagnards laissant un cortège d'accumulations glaciaires particulièrement frais (stade 4 ou « stade des cirques »), avant leur disparition 
définitive. Les dépôts correspondent à des moraines frontales, organisées dans l'espace de manière complexe. En effet, au sein d'un cirque, des constructions morainiques d'âge proche peuvent être juxtaposées (moraines de glacier de paroi et de glacier de cirque). Cependant, au-delà de ces différences, trois cirques présentent deux cordons morainiques bien individualisés, $4 \mathrm{a}$ et $4 \mathrm{~b}$ (fig. 1 , tab. 1) : ceux du Schiessrothried dans la vallée de la Wormsa, de Frankenthal et de Misseihmle dans le bassin de la Petite Fecht.

\section{Un complexe latéro-frontal clé : les moraines du stade 3 de la Wormsa}

La vallée de la Wormsa constitue l'un des plus beaux exemples parmi les systèmes glaciaires des Hautes Vosges. Aussi, ses héritages morainiques ont-ils fait l'objet de diverses études (Mercier et al. 1999 ; Jeser, 2000 ; Flageollet, 2002 ; Schmidt, 2002 ; Andreoli, 2003). Nous reprenons ici nos principaux résultats des analyses lithostratigraphiques et sédimentologiques conduites sur un complexe latéro-frontal contemporain du stade 3 , qui apportent un éclairage précis sur le rythme de la déglaciation.

\section{Contexte géomorphologique}

La vallée de la Wormsa, affluente de rive gauche de la Grande Fecht en amont de Metzeral, est ouverte dans le flanc sud du massif du Hohneck (1 363 m). La basse vallée comporte un ensemble complexe d'héritages morainiques et fluvioglaciaires appartenant aux stades 2 et 3 (fig. 3). Les moraines du stade 2 ferment la vallée de la Wormsa à sa confluence avec la vallée de la Grande Fecht. Le dispositif se compose, comme à Erbersch, de trois arcs morainiques successifs. Le premier (2a) forme un vaste lobe au débouché du vallon sur la Grande Fecht. En amont de ce dernier, deux arcs morainiques résiduels s'individualisent dans le paysage ( 2 b et $2 c$ ). Ces dispositifs frontaux s'associent latéralement à des faisceaux morainiques juxtaglaciaires empâtant le pied des versants.

Le complexe morainique du stade 3 de la Wormsa se développe directement en amont des moraines du stade 2 . Deux vallums morainiques terminaux se succèdent rapidement. La moraine frontale $3 \mathrm{a}$, culminant à $15 \mathrm{~m}$ au-dessus du fond de la vallée, constitue un barrage à convexité tournée vers l'aval, repoussant la Wormsabachrunz sur sa rive droite. Elle est associée, par ailleurs, à un petit cône proglaciaire. Ajoutons que l'ensemble du dispositif semble recouvrir des dépôts morainiques antérieurs (moraines 2c). La

\begin{tabular}{|c|c|c|c|c|c|c|c|c|c|c|c|c|c|}
\hline & \multicolumn{7}{|c|}{ Bassin de la Petite Fecht } & \multicolumn{6}{|c|}{ Bassin de la Grande Fecht } \\
\hline & \multicolumn{3}{|c|}{ Petite Fecht } & \multirow[t]{2}{*}{ Schlucht } & \multicolumn{3}{|c|}{ Altenbach } & \multirow[t]{2}{*}{$\begin{array}{c}\text { Grande } \\
\text { Fecht }\end{array}$} & \multicolumn{2}{|c|}{ Wormsa } & \multirow[t]{2}{*}{ KolbenFecht } & \multirow[t]{2}{*}{ Seestaedtle } & \multirow[t]{2}{*}{ Wiedenbach } \\
\hline & Fr N & Fr F & GD & & $M$ & $\mathrm{~B}$ & $\mathrm{CM}$ & & Sch & Fis & & & \\
\hline \multirow{4}{*}{ Stade 4} & $\Delta$ & $4 \mathrm{a} 1$ & \multirow{4}{*}{4} & \multirow{4}{*}{4} & \multirow{2}{*}{$4 a$} & \multirow{4}{*}{4} & \multirow{4}{*}{4} & \multirow{4}{*}{$?$} & \multirow{2}{*}{$4 a$} & \multirow{4}{*}{$?$} & \multirow{4}{*}{4} & \multirow[t]{2}{*}{$4 a$} & \multirow{4}{*}{4} \\
\hline & $4 a$ & $4 \mathrm{a} 2$ & & & & & & & & & & & \\
\hline & \multirow{2}{*}{$4 b$} & $4 b 1$ & & & $4 \mathrm{~b}$ & & & & $4 \mathrm{~b}$ & & & \multirow{2}{*}{$4 b$} & \\
\hline & & $4 \mathrm{~b} 2$ & & & & & & & $4 \mathrm{~N}$ & & & & \\
\hline \multirow{4}{*}{ Stade 3} & & 3a1 & & \multirow{2}{*}{ 3a1 } & \multirow{2}{*}{\multicolumn{3}{|c|}{3}} & \multirow{4}{*}{3} & \multirow{2}{*}{\multicolumn{2}{|c|}{ 3a }} & \multirow{2}{*}{$3 a$} & \multirow{2}{*}{$3 a$} & \multirow{4}{*}{3} \\
\hline & & $3 a 2$ & & & & & & & & & & & \\
\hline & & $3 b 1$ & & $3 b 1$ & & & & & 21 & & $2 h$ & $2 h$ & \\
\hline & & $3 b 2$ & & $3 b 2$ & & & & & Ut & & 30 & 30 & \\
\hline & & & $a$ & & & & & & 2 & & & $2 a$ & \\
\hline Stade 2 & & & $b$ & & & 2 & & 2 & 21 & & & $2 b$ & \\
\hline & & & $c$ & & & & & & 2 & & & $2 c$ & \\
\hline IDMG & & & & 1 & & & & & & & $1 \mathrm{a}$ & & \\
\hline Jivich a s & & & & 1 & & & & & & & $1 \mathrm{~b}$ & & \\
\hline & & & & $\mathrm{Mw}$ & & & & & & & $\mathrm{Mw}$ & & \\
\hline Munctor & & & & Mwa & & & & & & & Mwa & & \\
\hline של & & & & Mwb & & & & & & & Mwb & & \\
\hline & & & & & & & & & & & Mwc & & \\
\hline
\end{tabular}

Abréviations : Fr N : Frankenthal Nord ; Fr F : Frankenthal Falimont ; GD : Grotte Dagobert ; M : Missheimle ; B : Baerenbach ; CM : Cote 1000 ; Sch : Schiessrothried ; Fis : Fischboedle ; $M w(a, b, c)$ : moraines attribuées au Würm moyen.

Tableau 1 - Corrélation entre les moraines des stades glaciaires dans les bassins de la Grande Fecht et de la Petite Fecht. Table 1 - Correlation between terminal moraines of each stage in the drainage basins of La Grande Fecht and La petite Fecht. 
moraine frontale $3 b$ est beaucoup moins imposante ( $3 \mathrm{~m}$ de hauteur) mais forme un cordon quasi continu, rompu en son milieu par l'entaille de la rivière. La cuvette terminale comprise entre les deux constructions morainiques frontales est colmatée par un remblaiement glaciaire et fluvioglaciaire, plaqué en aval sur le flanc interne de la moraine $3 \mathrm{a}$.

\section{Lithostratigraphie du remblaiement}

Une coupe, haute de 2,5 m, est ouverte dans cette accumulation glaciaire et fluvioglaciaire. La base de l'affleurement se situe au niveau de la nappe alluviale actuelle. Les trois profils décrits (fig. 4A, B et C) donnent une image complète de la succession lithostratigraphique.

Ce remblaiement glaciaire et fluvioglaciaire a fait l'objet d'une analyse granulométrique limitée, compte tenu des propriétés texturales du dépôt, à la fraction sableuse. Cette analyse permet de proposer une typologie des sédiments reposant sur l'analyse des données sédimentologiques et granulométriques associées aux caractères morphométriques et géométriques des dépôts relevés sur le terrain (tab. 2). Cette typologie prend aussi en compte deux paramètres statistiques (fig. 5) : la médiane, qui exprime le calibre moyen des sables, et l'indice de tri (Folk et Ward, 1957) qui donne des indications sur les actions de sélection exercées sur le sédiment au cours du transport et du dépôt. Les résultats obtenus s'accordent clairement avec les caractéristiques spécifiques des courbes cumulatives de la phase sableuse (fig. 6). L'analyse conjointe de ces critères permet ainsi de proposer quatre classes de sédiments type 1, till s.s. ; type 2, alluvions fluvioglaciaires grossières relevant d'une dynamique de sédimentation peu sélective et tributaires d'écoulements à forte variabilité de débit ; type 3, alluvions fluvioglaciaires fines issues d'écoulements peu compétents mais sélectifs ; type 4, dépôts glacio-lacustres $s . l$. spécifiques

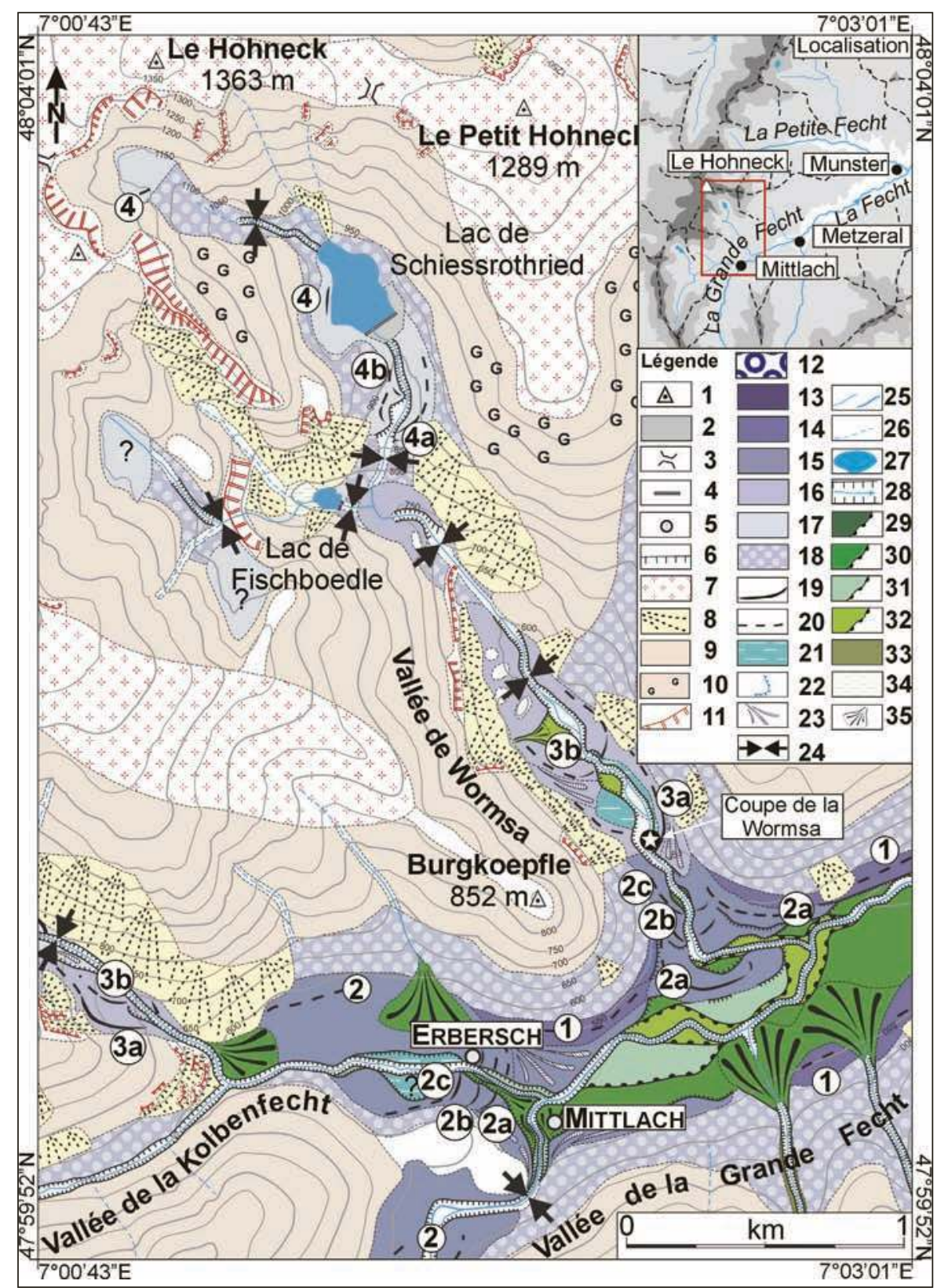

Fig. 3 - Carte des formations superficielles de la vallée de la Wormsa (Vosges, France). 1 : sommet $; 2$ : remblais anthropiques $; 3: \mathrm{col} ; 4$ : barrage $; 5:$ localité $; 6:$ talus $; 7:$ arènes granitiques ; 8 : éboulis à gros blocs $; 9$ : formations de pente périglaciaires indéterminées ou polygéniques ; 10 : gélifluxion ; 11 : escarpement $; 12$ : moraines « rissiennes »; 13 : moraines du Würm moyen ; 14 : moraine (stade 1) ; 15 : moraines (stade 2) ; 16 : moraines (stade 3) ; 17 : moraines (stade 4) ; 18 : dépôt morainique remanié ; 19 : ride morainique ; 20 : cordon morainique discontinu ; 21 : dépôt fluvioglaciaire et glaciolacustre de retrait ; 22 : dépôt d'obturation latérale, gradin latéral ou kame ; 23 : cône proglaciaire ; 24 : verrou ; 25 : cours d'eau pérenne ; 26 cours d'eau intermittent ; 27 : lac, étang ; 28 : lit majeur actuel ; 29 : moyenne terrasse supérieure $\mathrm{T} 1 \mathrm{a} ; 30$ : moyenne terrasse intermédiaire $\mathrm{T} 1 \mathrm{~b} ; 31$ : moyenne terrasse inférieure T1c ; 32 : basse terrasse postglaciaire T0 ; 33 : dépôt torrentiel ; 34 : tourbière, marais ; 35 : cône de déjection.

Fig. 3 - Map of the Wormsa valley field (Vosges, France). 1: summit; 2: backfill; 3: pass; 4: dam; 5: locality; 6: bank; 7: grus; 8: bouldery talus; 9: undetermined or polygenetic periglacial deposits; 10: gelifluxion; 11: steep slope; 12: till (Riss); 13: till (Middle Würm); 14: stage 1 till; 15: stage 2 till; 16: stage 3 till; 17: stage 4 till; 18: reworked till; 19: morainic ridge; 20: discontinuous morainic ridge; 21: fluvioglacial and glaciolacustrine deposits indicating ice retreat; 22: icecontact valley-side terrace or kame delta; 23: proglacial fan; 24: rock-bar; 25: river; 26 ephemeral stream; 27: lake, pond; 28: present-day river bed; 29: fluvioglacial terrace T1a; 30: fluvioglacial terrace T1b; 31: fluvioglacial terrace T1c; 32: postglacial terrace T0; 33: torrential deposits; 34: peat bog; 35: alluvial or torrential fan. 


\begin{tabular}{|c|c|c|c|c|c|}
\hline Type & $\begin{array}{l}\text { Fraction > } \\
50 \mu \mathrm{m}(\%)\end{array}$ & $\begin{array}{l}\text { Fraction < } \\
50 \mu \mathrm{m}(\%)\end{array}$ & Médiane $(\mathrm{mm})$ & $\begin{array}{l}\text { Indice de tri } \\
\text { so }\end{array}$ & Description \\
\hline 1 & {$[60-75]$} & {$[40-25]$} & {$[0.4-0.5]$} & {$[1.382 ; 1.524]$} & $\begin{array}{l}\text { Formations caillouteuses sans stratification } \\
\text { apparente à galets sains ou peu altérés, } \\
\text { émoussés à sub-anguleux de taille centimétrique } \\
\text { à pluridécimétrique. }\end{array}$ \\
\hline 2 & {$[79-98]$} & {$[2-21]$} & {$[0.4-1.0]$} & {$[0.913 ; 1.361]$} & $\begin{array}{l}\text { Dépôts sablo-graveleux riches en galets } \\
\text { hétérométriques présentant in situ des amorces } \\
\text { de stratification et de tri du matériel. }\end{array}$ \\
\hline 3 & {$[65-98]$} & {$[2-35]$} & {$[0.1-0.6]$} & {$[0.525 ; 1.122]$} & $\begin{array}{l}\text { Les dépôts présentant des structures } \\
\text { sédimentaires assez nettes (stratification plane } \\
\text { ou lenticulaire). }\end{array}$ \\
\hline 4 & {$[16-58]$} & {$[42-84]$} & {$[0.07-0.20]$} & 0.413 et 0.870 & $\begin{array}{l}\text { Dépôts organisés selon des plans de } \\
\text { stratification perturbés par des plications et des } \\
\text { involutions noyant des galets ou blocs isolés, de } \\
\text { taille centimétrique à décimétrique. }\end{array}$ \\
\hline
\end{tabular}

Tableau 2 - Caractéristiques granulométrique et stratigraphique par type de sédiments.

Table 2 - Grain size distribution and stratigraphic characteristics for each type of sediments.

de dépôts de décantation, typiques de zones de sédimentation en eau calme.

La coupe présente deux séquences sédimentaires $\mathrm{S} 1$ et $\mathrm{S} 2$ séparées par une discontinuité érosive (fig. 4). La séquence S1 (fig. 4, profil C) associe, sur $60 \mathrm{~cm}$ d'épaisseur, des termes sableux à graveleux avec des passées limoneuses disposés selon une stratification grossièrement plane. Ces dépôts sont pauvres en éléments grossiers ; seuls quelques galets, toujours inférieurs à $10 \mathrm{~cm}$, ont été décelés dans le lit de sables grossiers et de graviers qui coiffe l'unité. Les échantillons prélevés dans cette séquence appartiennent aux types 2 et 3 et mettent en évidence le caractère fluvioglaciaire du dépôt.

La séquence $\mathrm{S} 2 \mathrm{a}$ (fig. 4, profils B et $\mathrm{C}$ ) constitue un dépôt d'environ un mètre d'épaisseur, très hétérométrique, à galets de granites émoussés à sub-anguleux de taille centimétrique à pluridécimétrique, emballés dans une matrice sableuse à sablo-limoneuse abondante. La formation comporte de petites poches ou « galets» de sables organiques. Les échantillons issus de ce niveau appartiennent tous au type 1 de till s.s.

La séquence $\mathrm{S} 2 \mathrm{~b}$ (fig. 4, profils A et $\mathrm{B}$ ) associe des lits millimétriques de limons et de sables fins organisés en fines strates horizontales à sub-horizontales présentant des traces d'hydromorphie, et des lentilles caillouteuses à matrice sablo-graveleuse disposées en stratification oblique. Les termes limoneux et sableux enveloppent des galets de granites émoussés, profondément arénisés, de taille centimétrique à pluricentimétrique, qui perturbent le litage. Dans le détail, la stratification est aussi dérangée par des plications formant parfois des involutions. Deux coupes complémentaires montrent que cette unité se prolonge vers le haut, jusqu'au sommet de la formation (Andreoli, 2003). L'analyse granulométrique des échantillons prélevés dans ce niveau montre que ce dépôt associe des apports fluvioglaciaires (types 2 et 3 ) et des sédiments glacio-lacustres s.l. (type 4).

\section{Interprétation des résultats}

D'après M. Schmidt (2002), les dépôts fluvioglaciaires de la séquence S1 peuvent avoir deux origines : des écoulements sous-glaciaires ou des écoulements proglaciaires. L'enchaînement lithostratigraphique observé à la base du remblaiement, entre $\mathrm{S} 1$ et $\mathrm{S} 2 \mathrm{a}$, nous fait accréditer la seconde hypothèse : les alluvions fluvioglaciaires sont en effet tronquées en biseau par les dépôts morainiques. Le phénomène s'accorde avec le scénario suivant : édification d'un sandur proglaciaire par les eaux de fusion qui s'échappaient d'un front positionné en amont, puis avancée du front glaciaire sur le site, responsable du dépôt morainique de la séquence $\mathrm{S} 2 \mathrm{a}$.

Fig. 4 - Coupe des formations glaciaires et fluvio-glaciaires de la Wormsa. 1 : dépôt morainique ; 2 : galets et blocs émoussés ; 3 : galets anguleux $; 4$ : sables grossiers, graviers $; 5$ : sables $; 6$ : sables fins laminés ; 7 : limons ou limons argileux compacts ; 8 : limons ou limons argileux laminés ; 9 : discontinuité stratigraphique ; $10:$ sol en place.

Fig. 4 - Glacial and fluvioglacial deposits of stage 3 in the Wormsa valley. 1: till; 2: subrounded pebbles and boulders; 3 : angular pebbles; 4: sand and gravel; 5: sands; 6: laminated silty sand; 7: silt and clay; 8: laminated silt and clay; 9: stratigraphic unconformity; 10: soil. 


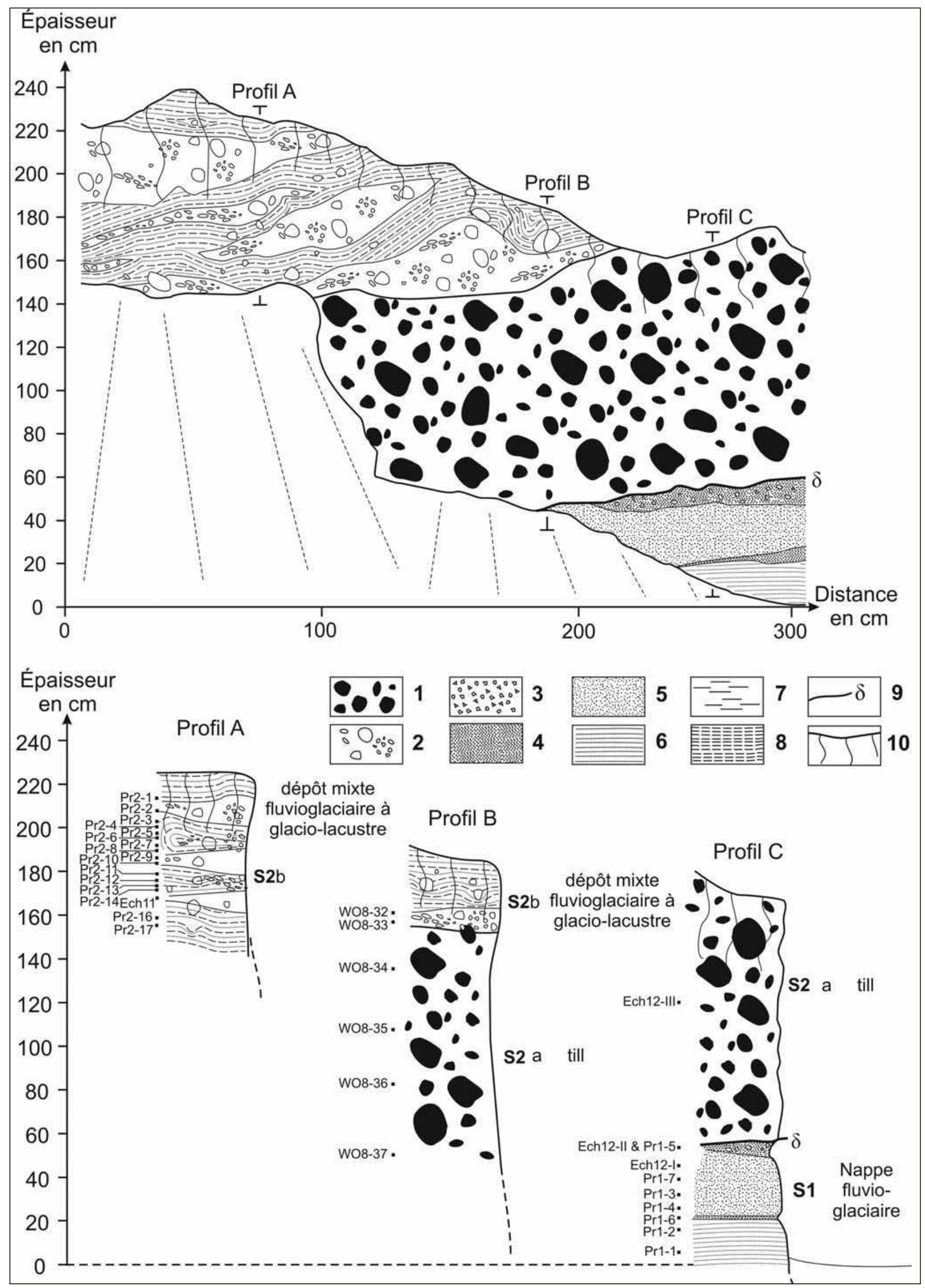


Fig. 5 - Typologie des sédiments selon le critère de tri granulométrique.

Fig. 5 - Typology of sediments based on sorting index.

Des alluvions fluvioglaciaires sableuses (type 3) interstratifiées avec des dépôts glacio-lacustres (type 4), caractérisant l'essentiel de la séquence $\mathrm{S} 2 \mathrm{~b}$, doivent être mises en relation avec une plaine d'épandage globalement mal drainée, obstruée en aval par la moraine terminale du stade 3a. Les apports strictement fluviatiles étaient restreints à des chenaux proglaciaires peu puissants (type 3 ) et cohabitaient avec des apports diffus de fines, décantées dans des flaques d'eau stagnantes (type 4). Les galets de granites épars, perturbant les lits de sables et de limons correspondent, soit à des dropstones charriés par des micro radeaux de glace et piégés lors de la fonte au fond des étendues d'eau stagnantes, soit à des effondrements de blocs issus de l'arc morainique surplombant la plaine d'épandage. Enfin, les lentilles caillouteuses (types 2 et 3) dont la stratification est oblique dans les lits limono-sableux sont très probablement liées à des chenaux proglaciaires puissants et mobiles, actifs lors des crues. Enfin, la partie sommitale de la séquence fluvioglaciaire S2b, visible sur deux autres coupes, met en évidence des apports limoneux non triés et une concentration plus importante en blocs (Andreoli, 2003). Ces faciès semblent refléter des événements plus brutaux déclenchés par la fonte accélérée du glacier (dépôts de boue limoneuse survenant lors de crues importantes ; dropstones liés à la débâcle glaciaire).

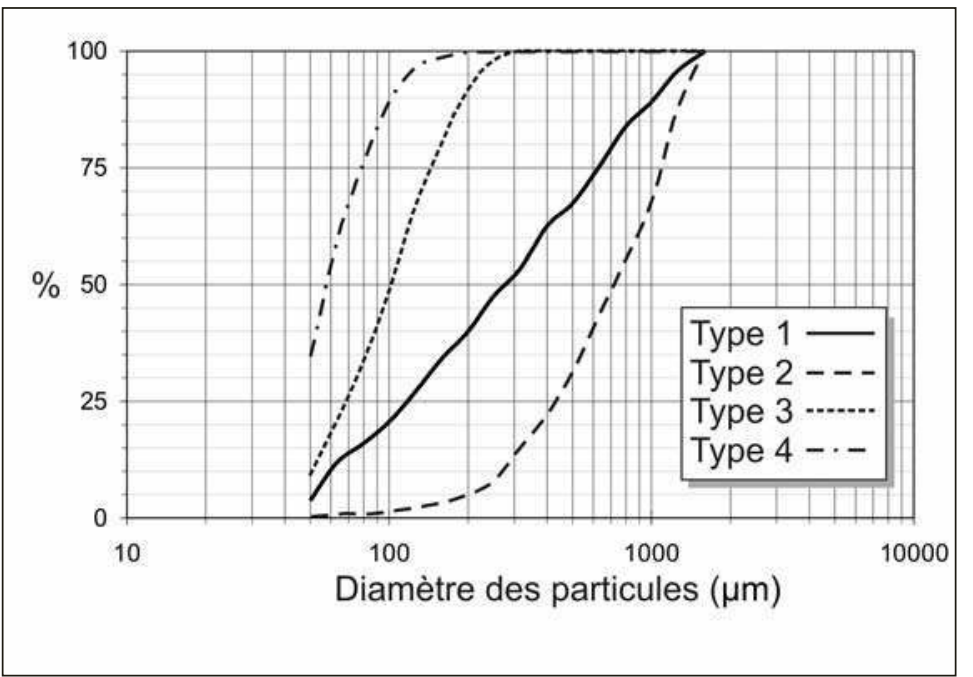

Fig. 6 - Courbe cumulative de référence de la fraction sableuse par type de sédiments.

Fig. 6 - Reference cumulative curve of sand part for each sediment type.

\section{Essai de chronologie relative}

L'analyse sédimentologique et stratigraphique du remblaiement glaciaire et fluvioglaciaire du stade 3 de la Wormsa apporte un éclairage précis sur le déroulement de la déglaciation dans ce secteur de la vallée (fig. 7). La dynamique globale de retrait du glacier de la Wormsa vers sa haute vallée est, semble-t-il, interrompue par un épisode de récurrence glaciaire, dont témoigne encore dans le paysage actuel le vallum morainique frontal du stade $3 \mathrm{a}$.

Une première phase de récession glaciaire succède au stade 2c et s'accompagne du dépôt des alluvions proglaciaires de la séquence S1 (sandur de retrait). L'amplitude du retrait reste difficile à préciser ; notons cependant que l'absence d'alluvions grossières (blocs et galets) dans la séquence fluvioglaciaire suggère un éloignement du front vers le haut bassin de la Wormsa (fig. 7A). Une réavancée glaciaire (stade $3 \mathrm{a}$ ) succède à la récession précédente. La formation morainique $\mathrm{S} 2 \mathrm{a}$ qui signe cet événement semble en relation directe avec la moraine frontale $3 \mathrm{a}$. L'absence de lessivage ou d'amorce de tri dans le dépôt, l'absence de glacitectonique dans les alluvions sous-jacentes et la morphologie du complexe morainique frontal suggèrent une réavancée glaciaire brève sur un sol gelé (fig. 7B). Un début de retrait glaciaire s'amorce ensuite dans le secteur de la basse vallée : les flux hydrosédimentaires nourris par la décrue glaciaire sont piégés dans la cuvette terminale barrée en aval par l'arc morainique $3 \mathrm{a}$ (fig. 7C). Un stade glaciaire 3b, concomitant semble-t-il des apports limoneux à forte concentration en blocs qui coiffent le dépôt fluvioglaciaire $\mathrm{S} 2 \mathrm{~b}$, traduit l'occurrence de phénomènes violents (crues de ruptures d'embâcles glaciaires ? Andreoli, 2003). Au total, l'ensemble du remblaiement fluvio- 

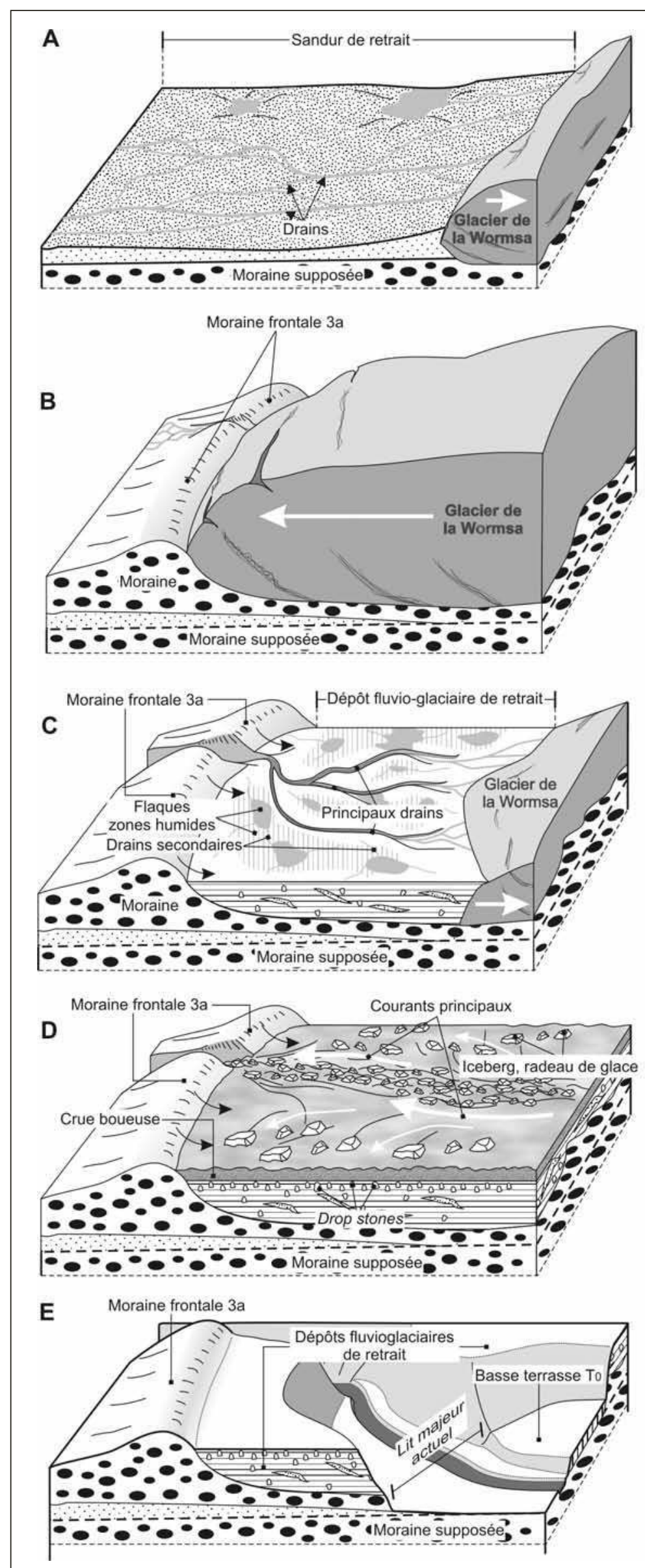

Fig. 7 - Essai de chronologie des formations du stade 3a de la Wormsa.

Fig. 7 - Tentative chronology of the 3a stadial in the Wormsa valley.

glaciaire constitue aujourd'hui une formation au modelé ambigu, raccordée en amont au vallum morainique frontal 3b (fig. 7D). Enfin, la séquence chronologique s'achève par le retrait définitif de la langue glaciaire vers les cirques du haut bassin. L'incision qui a affecté l'ensemble des dépôts morainiques et fluvioglaciaires est liée, sans doute dans une large mesure, aux eaux de fusion gonflées par l'importante décrue glaciaire qui succède au stade $3 b$. Au cours du Postglaciaire, le dispositif glaciaire résiduel a constitué un piège favorable au remblaiement de la basse terrasse $\mathrm{T}_{0}$ (fig. $7 \mathrm{E}$ ).

\section{Discussion}

Outre des placages lacunaires, peu caractéristiques et mal situés stratigraphiquement, l'essentiel des complexes morainiques du haut bassin de la Fecht semble se rattacher au Pléniglaciaire würmien. Leurs caractéristiques géométriques et sédimentologiques plaident en faveur de deux phases d'englacement distinctes. Une première progression glaciaire, apogéique, est responsable des accumulations morainiques latérales des sections moyennes des vallées (groupe IIa). À son maximum d'extension, le glacier de la Fecht s'avançait largement dans la moyenne vallée en direction de Munster (Krittlé, 1956). Cependant, l'extension et les caractéristiques de l'englacement sont encore difficiles à cerner ; les études antérieures admettent l'hypothèse d'une calotte glaciaire située sur la crête principale, émettant plusieurs langues glaciaires de vallées effluentes (Flageollet, 2002 ; Mercier et Jeser 2001 ; Seret, 1967, 1990). Selon C. Krittlé (1956, 1958) et J. Tricart (1963), cet épisode doit être rattaché à l'avant-dernière glaciation (« Riss »). Mais les analogies sédimentologiques et stratigraphiques des héritages glaciaires du bassin de la Fecht et des dispositifs morainiques décrits par les auteurs sur le versant occidental du massif vosgien (Seret, 1967, 1990), comme les observations réalisées par N. Jeser (2000) et J.-L. Mercier et N. Jeser $(2001,2004)$, conduisent à attribuer cette phase de progression glaciaire au Würm moyen.

La dernière phase d'englacement est soulignée par les complexes morainiques latérofrontaux des sections amont des principales vallées du haut bassin de la Fecht (groupe IIb). 
L'homogénéité morphologique et la continuité géométrique du dispositif démontrent son unité stratigraphique. Les héritages glaciaires du secteur de Metzeral, situés le plus en aval, en constituent les « moraines du maximum ». Pour C. Krittlé (1956, 1958), J. Tricart (1963) et J.-C. Flageollet (2002), celles-ci signent l'extension maximale des glaces au cours du Würm. Sans entrer dans ce débat, il faut admettre que le dispositif glaciaire de Metzeral correspond au Dernier Maximum Glaciaire (DMG) du bassin de la Grande Fecht. Les caractéristiques morphologiques des dépôts morainiques du groupe IIb témoignent de glaciers de vallée de type alpin, individualisés dans des auges profondes qu'ils n'occupaient pas en totalité. Plusieurs appareils peuvent être clairement identifiés (fig. 1) : langues de glace des vallées de la Wormsa (massif du Hohneck) et de la Grande Fecht ; langues effluentes du bassin de la Kolbenfecht, issues de cirques ouverts sous les crêtes du Kastelberg, Rainkopf, Batteriekopf et Rothenbachkopf. Les trois courants de glace principaux s'unifiaient en aval pour former un glacier de vallée unique, dont l'extension maximale au DMG (« stade de Metzeral ») ne dépassait pas sept kilomètres depuis la crête faîtière.

\section{Les grandes étapes de la récession glaciaire dans le haut bassin de la Fecht}

Après l'épisode du « stade de Metzeral », le retrait glaciaire s'est effectué en trois grandes étapes (tab. 1). Le premier épisode de la déglaciation s'accompagne d'une première individualisation des glaciers. Il précède une phase de stationnement majeure, clairement enregistrée dans les dispositifs morainiques frontaux du stade 2 (« Stade de Erbersch »). Dans le bassin de la Grande Fecht, les glaciers de la Grande Fecht de Mittlach, de la Kolbenfecht et de la Wormsa s'individualisent dans leurs vallées, établissant leur front à quelques kilomètres en amont de Metzeral. Après le stade 2, la récession glaciaire semble être de forte ampleur, comme le suggèrent les données morpho-stratigraphiques réunies dans la vallée de la Wormsa. Dans ce contexte, les moraines du stade 3 ( «Stade de Seestaedtle ») témoignent d'un épisode bref de réavancée des langues de glace qui remet en cause le mouvement global de décrue. La récurrence glaciaire correspond ainsi à une dernière avancée des glaciers avant la déglaciation définitive des vallées et leur retrait dans les cirques d'altitude. La disparition des langues glaciaires a provoqué une déstabilisation des versants libérés des glaces à cause de la décompression, responsable de la mise en place de vastes tabliers et cônes d'éboulis (Andreoli, 2003). Enfin, les glaciers ont persisté en position d'abri dans les cirques glaciaires des hauts bassins montagnards, laissant un cortège d'accumulations morainiques particulièrement frais et complexe (stade 4 ou stade des cirques) dont l'interprétation chronologique et morphodynamique nous échappe encore dans une large mesure.

En l'absence de datation absolue, la chronologie présentée reste relative. Aussi, plusieurs interrogations subsistent. Ces dernières concernent notamment l'âge de la déglaciation définitive du bassin de la Fecht et, au-delà, du massif vosgien. Les études palynologiques effectuées sur différentes tourbières du massif permettent d'apporter quelques éléments de réflexion. L'enregistrement pollinique de la tourbière de la Grande Pile (versant Sud des Vosges), quasi continu sur l'ensemble du dernier cycle bioclimatique, fait état d'un dernier maximum de froid compris entre $25 \mathrm{ka}$ et $15 \mathrm{ka}$ BP ; puis un retrait glaciaire débutant vers 15 ka BP (Woillard, 1978, 1979 ; Woillard et Mook, 1982). De plus, les spectres polliniques des tourbières de fond de vallée ne débutent jamais avant 12 ka BP (vallée d'Urbès affluente de la Thur ; Firbas et al, 1948). Enfin, dans les remplissages tourbeux des cirques et ombilics des hauts bassins, les séquences palynologiques sont toujours postérieures à $9 \mathrm{ka}$ BP (Hatt, 1937 ; Lemée, 1963 ; Tricart, 1963 ; Oschenbein, 1977 ; Flageollet, 2002). Ces données offrent l'image d'une déglaciation tardive des vallées des hauts bassins vosgiens. De ce fait, certains auteurs avancent l'hypothèse de glaciers et névés dans les cirques jusqu'au Dryas récent (11-10 ka BP ; Lemée, 1963 ; Tricart, 1963 ; Darmois-Theobald et al., 1976). Par comparaison, dans le Jura, massif voisin des Vosges et aux altitudes similaires, le retrait glaciaire définitif se situerait vers $17 \mathrm{ka} \mathrm{BP}$ (Buoncristiani et al., 2002). Les résultats récents des analyses par isotopes cosmogéniques au ${ }^{10} \mathrm{Be}$ de roches moutonnées ou blocs erratiques associés aux dispositifs morainiques frontaux de la vallée de la Wormsa, présentés par J.-L. Mercier et al. (1999), apportent de nouveaux éléments au débat. Outre qu'ils confirment largement le caractère tardif de la déglaciation vosgienne, ils proposent un cadre chronologique particulièrement original. Selon ces travaux, en effet, la récession glaciaire du bassin de la Grande Fecht se serait déroulée sur cinq millénaires, de la fin du Tardiglaciaire au début de l'Holocène : cinq stades de retrait sont ainsi identifiés à 11,5 $\pm 1 \mathrm{ka}, 10,6 \pm 1,1 \mathrm{ka}$, $9,7 \pm 1,1 \mathrm{ka}, 9 \pm 1,1 \mathrm{ka}, 8,3 \pm 1,1 \mathrm{ka}{ }^{10} \mathrm{Be} \mathrm{BP}$, plaçant ainsi les moraines du stade de Erbersch (stade 2) au Tardiglaciaire supérieur et celles du stade 3 (stade de Seestaedtle) au Boréal. Par ailleurs, la déglaciation définitive des cirques d'altitude appartiendrait à la période Atlantique ; les deux dates obtenues sur roches moutonnées étant les suivantes : $5,1 \pm 1,2 \mathrm{ka}{ }^{10} \mathrm{Be}$ ans BP et $6,3 \pm 0,8 \mathrm{ka}{ }^{10} \mathrm{Be}$ ans BP. Il faut noter que ces données singularisent nettement le versant alsacien des Vosges par rapport aux autres massifs montagnards d'Europe occidentale où les appareils glaciaires, lorsqu'ils subsistent encore, se trouvent partout cantonnés aux hauts bassins montagnards dès le début du Tardiglaciaire.

\section{Conclusion}

La chronologie relative proposée apporte un nouvel éclairage sur les modalités de la dernière phase d'englacement et le rythme du retrait glaciaire dans le haut bassin de la Fecht. Après le maximum de progression glaciaire de Metzeral, la déglaciation, réalisée en trois grandes étapes, est interrompue, au moins localement dans la vallée de la Wormsa, par une phase de réavancée glaciaire. Ce schéma stratigraphique 
et morphodynamique local doit être replacé dans son contexte régional. Cependant, les corrélations que nous pouvons aujourd'hui envisager reposent sur des bases incertaines. En effet, si les synthèses réalisées sur les systèmes glaciaires vosgiens offrent une image assez précise des caractéristiques de l'englacement du massif, en particulier sur sa retombée occidentale (Seret, 1967 ; Seret et al., 1990 ; Jeser, 2000 ; Mercier et Jeser, 2001 et 2004 ; Flageollet, 2002), les conclusions des auteurs sont parfois divergentes. Les discussions portent notamment sur le nombre et les limites d'extension des différents appareils glaciaires pléistocènes et sur la position du Dernier Maximum Glaciaire (DMG). D'autre part, les données stratigraphiques recueillies dans les vallées du versant alsacien des Vosges sont encore incomplètes. Tel est le cas du haut bassin de la Fecht, où l'extension maximale des glaces au Quaternaire est méconnue et la position du maximum d'englacement au Pléniglaciaire « würmien» est encore incertaine. Enfin, et plus généralement, la comparaison des deux versants du massif vosgien s'avère hasardeuse, car elle oppose deux domaines possédant des caractéristiques paléogéomorphologiques et topoclimatiques très différentes.

Dès lors, les perspectives de nos recherches sont doubles. Nous comptons dépasser le cadre stratigraphique local obtenu dans cette étude sur la base d'une comparaison entre les stades de retrait du haut bassin de la Fecht et les dispositifs morainiques d'autres secteurs clés du massif vosgien (vallées de la Thur, de la Doller et haute vallée de la Fecht $s . s$ sur le versant oriental des Vosges ; Moselotte sur son versant occidental). Nous souhaitons obtenir de nouvelles datations absolues en complément des résultats des analyses cosmogéniques effectuées par J.-L. Mercier et al. (1999).

\section{Remerciements}

Nous tenons à remercier Stephen Clandillon pour avoir consacré un peu de son temps à la relecture des textes en anglais.

\section{Références}

Andreoli R. (2003) - Le haut bassin de la Fecht du dernier glaciaire au postglaciaire : bilan des travaux, nouvelles données, et perspectives. Mémoire de DEA, université L. Pasteur Strasbourg, $128 \mathrm{p}$.

Baulig H. (1922) - Questions de morphologie vosgienne et rhénane (Deuxième article). Annales de Géographie, 173, XXXI, 385-401.

Bourlès D., Benedetti L., Braucher R. (2004) - Rayons cosmiques et chronologie des paysages. Pour la Science, 24, 66-69.

Buoncristiani J.-F., Campy M., Pugin A. (2002) - Modalité de la dernière extension glaciaire maximale dans le Jura et proposition de corrélation avec l'enregistrement isotopique du Groenland. In Richard H. et Vignot A. (eds.), Équilibres et ruptures dans les écosystèmes durant les 20 derniers millénaires en Europe de l'Ouest, Actes du colloque international de Besançon, septembre 2000. Presses Universitaires Franc-comtoises, 27-34.
Darmois-Théobald M., Denefle M., Ménillet F. (1976) - Tourbière de moyenne altitude de la forêt de la haute Meurthe (Vosges, France). Bulletin de l'Association française pour l'étude du Quaternaires, 2, 99-107.

Firbas F., Grünig G., Weischedel I., Worzel G. (1948) - Beiträge zur spät und nacheiszeitlichen Vegetationsgeschichte der Vogesen. Bibliotheca Botanica, 121, 1-76.

Flageollet J.-C. (2002) - Sur les traces des glaciers vosgiens. CNRS éditions, Paris, 212 p.

Folk R.L., Ward W.C. (1957) - Brazos River bar: a study in the significance of grain size parameters. Journal of Sedimentary Petrology, 27, 3-26.

Hatt J.P. (1937) - Contribution à l'analyse pollinique des tourbières du Nord-Est de la France. Bulletin du Service des Cartes géologiques d'Alsace-Lorraine, T. 4, 1-77.

Jeser N. (2000) - Les grandes étapes du glaciaire Würmien dans les Vosges (cartographie et analyse). Mémoire de DEA, université L. Pasteur Strasbourg, 119 p.

Krittlé C. (1956) - Étude cartographique des phénomènes glaciaires et fluviatiles de la vallée de la Fecht jusqu'à Turkheim. DES de géologie, université L. Pasteur Strasbourg, 120 p.

Krittlé C. (1958) - Morphologie et dépôts quaternaires antérieurs au Würm dans la vallée de la Fecht (Haut-Rhin). Bulletin $d u$ Service des Cartes géologiques d'Alsace-Lorraine, T. 11, 15-19.

Lemée G. (1963) - L'évolution de la végétation et du climat des Hautes Vosges centrales depuis la dernière glaciation. In « Le Hohneck, aspects physiques, biologiques et humains », ouvrage collectif pour le centenaire de l'Association Philomatique d'Alsace et de Lorraine, Strasbourg, 185-192.

Menillet F., Blanalt J.G., Cloots A.R., Flageollet J.-C., Fluck P., Fourquin C., Gagny C., Hirlemann C., Lillie F., Ruhland M., Schumacher F., Stussi J.M., Trautmann J., Vogt H. (1976) Notice de la carte géologique $n^{\circ} 377$, Munster, BRGM.

Mercier J.L., Bourles D.L., Kalvoda I., Vergne V., Braucher R., Paschen A., Raisbeck G.M., Yiou F. (1999) - ${ }^{10}$ Be dating of moraines and roches moutonnées at low altitude in continental Europe during the Holocene. Geophysical Research Abstracts, 1,576 .

Mercier J.-L, Jeser N. (2001) - Cartography of the glacial past of the Vosges Mountains. Fifth international conference on Geomorphology, Tokyo, August 2001; abstracts of conferance papers, Transactions, Japanese Geomorphological Union. 22, 4, Page C-153

Mercier J.-L, Jeser N. (2004) - The glacial history of the Vosges mountains. In "Quaternary Glaciation - extent and chronology", Part I : Europe, Ehlers J., Gibbard P.L. (Eds.), Development in Quaternary Science, Vol. 2a, Amsterdam, Elsevier, 113-118.

Oschenbein G. (1977) - Reconstitution du tapis végétal après les glaciations. Saisons d'Alsace, n ${ }^{\circ}$ spécial 61-62, 22e année, 27-43.

Seret G. (1967) - Les systèmes glaciaires de la vallée de la Moselle et leurs enseignements. Société Royale Belge de Géographie, Bruxelles, $577 \mathrm{p}$.

Seret G., Dricot E., Wansard G. (1990) - Evidence for an early glacial maximum in the French Vosges during the last glacial cycle. Nature, 346, 453-456. 
Sittig G. (1933) - Topographie proglaciaire et topographie glaciaire des Vosges alsaciennes du sud. Annales de Géographie, 52, 248-265.

Schmidt M. (2002) - Étude sédimentologique de dépôts glaciaires sur le versant alsacien des Vosges. Mémoire de Maîtrise de géographie, université L. Pasteur Strasbourg, 87 p. + annexes.

Tricart J. (1963) - Aspects et problèmes géomorphologiques du massif du Hohneck, In « Le Hohneck, aspects physiques, biologiques et humains », ouvrage collectif pour le centenaire de l'Association Philomatique d'Alsace et de Lorraine, Strasbourg, ,43-62.
Woillard G. (1978) - Grande Pile peat bog: a continuous pollen record for the last 140000 years. Quaternary Research, 9, $1-21$.

Woillard G. (1979) - The last glacial-interglacial cycle at Grande Pile in northeastern France. Bulletin de la Société Belge de Géologie, 88, 51-69.

Woillard G. Mook W.G. (1982) - Carbon dates at Grande Pile: correlation of land sea chronologies. Science, 215, 159-161.

Article reçu le 19 décembre 2004, accepté le 9 décembre 2005. 